García Ramos, J. M., de la Calle Maldonado, C., Valbuena Martínez, M. C. y de Dios Alija, T. (2016). La formación en Responsabilidad Social y su impacto en diversas carreras universitarias. Revista de Investigación Educativa, 34(2), 435-451.

DOI: http://dx.doi.org/10.6018/rie.34.2.244271

\title{
La formación en Responsabilidad Social y su impacto en diversas carreras universitarias
}

\section{Educating in Social Responsibility and its impact on the students of a university's diverse degree courses}

*José Manuel García Ramos, ${ }^{* *}$ Carmen de la Calle Maldonado, ***aría Consuelo Valbuena Martínez y

** Teresa de Dios Alija

*Departamento de Métodos de Investigación y Diagnóstico en Educación.

Facultad de Educación. Universidad Complutense de Madrid (España)

**Universidad Francisco de Vitoria (España)

\begin{abstract}
Resumen
La Universidad Francisco de Vitoria (UFV) desarrolla desde 1993, el primer proyecto educativo en el ámbito europeo, que integra en sus planes de estudios una asignatura obligatoria para formar a sus alumnos en la responsabilidad social. Con esta formación pretendemos acercar al universitario al área de irradiación del compromiso social para que él mismo perciba el valor de lo que se le sugiere, lo asuma como propio y comprenda la importancia de plantearse el ejercicio socialmente responsable de su profesión. Nuestro propósito es evaluar el impacto de esta enseñanza en estudiantes universitarios. Para ello empleamos una herramienta fiable y válida, que mediante encuesta personal (muestra total: 757 estudiantes) facilita la recogida de información en dos momentos (pretest-postest). Los resultados indican que la asignatura de Responsabilidad Social tiene impacto significativo en los estudiantes, a nivel general y por dimensiones y es diferente en los distintos Grados universitarios.

Palabras clave: formación universitaria; responsabilidad social; estudiantes universitarios; impacto social.
\end{abstract}

Correspondencia: José Manuel García Ramos, Departamento MIDE. Facultad de Educación. C/Rector Royo Villanova, s/n. 28040-Madrid.E-mail: jmgramos@ucm.es 


\begin{abstract}
Since 1993, the Universidad Francisco de Vitoria (UFV) has developed the first educational project in Europe which integrates an obligatory subject across all university courses: the social responsibility. The aim of this course is to heighten the university student's awareness of social commitment and responsibility so that each individual gains an understanding of the ideas presented to him and subsequently take these values on board, making them his own and applying them to his future profession. Our aim is to evaluate the impact that this education has on our students. To do this, we have used a valid and reliable tool which, by means of a personal questionnaire, (sample size: 757 students), has allowed collect the information at two different points in time (pre-test and post-test). The results show that the Social Responsibility course has a significant impact on our students, and the effect varying depending on the degree being studied.

Keywords: university education; social responsibility; university students; social impact.
\end{abstract}

\title{
Introducción
}

La formación universitaria va más allá de ser una mera preparación técnica para el desempeño profesional, debe también contribuir a la formación integral de la persona. Por ello, en los planes de estudio de las titulaciones que se cursan en la Universidad Francisco de Vitoria incluimos un conjunto de materias de carácter humanístico orientadas a fomentar en el alumno una serie de valores, actitudes y hábitos, que le permitan en un futuro pensar con rigor, comprender y analizar de manera integral la realidad y poder tomar así las decisiones personales y profesionales también bajo el prisma de la responsabilidad ética.

Un universitario no puede dar la espalda a los problemas sociales de su entorno, ha de estar preparado para aportar soluciones concretas y comprometidas con el bien común desde el ejercicio de su profesión.

\section{Revisión teórica}

Este estudio se centra en el análisis del impacto de la formación en Responsabilidad Social del Estudiante universitario (RSEU) y no en la repercusión de la conocida como Responsabilidad Social Universitaria (RSU), más enfocada a la responsabilidad corporativa, institucional o empresarial (de Dios Alija, 2014).

Respecto a la primera hemos localizado estudios relacionados con impacto de la formación ética, como las aportaciones de Pascarella, Ethington y Smart (1998) que analizan la influencia de la universidad en la formación de valores humanitarios (Galán, Sáenz de Miera y de la Calle Maldonado, 2012); los efectos del voluntariado durante los años de estudiante (Astin, Sax y Avalos, 1999), el papel de la formación en la educación para la responsabilidad personal y social (Reason, Ryder y Kee, 2013) y la promoción de dones y talentos relacionados con el apoyo social y la Promoción Humanitaria (Hernández de Hahn, 2014).

En 2013 se presenta una amplia revisión de la literatura sobre el papel de la Educación Superior en la Educación para la Responsabilidad Personal y Social (Reason, Ryder, 
y Kee, 2013), de la que se pueden sacar interesantes conclusiones sobre el impacto del aprendizaje de la responsabilidad social en el desarrollo personal de los estudiantes en el contexto del cambio de educación superior (Sánchez González, Herrera Márquez, Zárate Moreno y Moreno Méndez, 2007).

En cuanto a la evaluación de la formación de la responsabilidad social del universitario, es necesario citar el análisis de eficacia de la asignatura de "Responsabilidad Social" en la UFV de cara a la formación integral de sus alumnos (de la Calle Maldonado, García Ramos, Giménez Armentia y Ortega de la Fuente, 2008), los trabajos de Larrán y colaboradores sobre la oferta y el impacto de asignaturas de responsabilidad social y ética empresarial en alguna titulaciones del ámbito de la gestión de organizaciones (Larrán Jorge y Andrades Peña, 2015), la cooperación para el desarrollo como metodología para la enseñanza de responsabilidad social de ingenieros (Lappalainen, 2011),y el análisis de oportunidades y desafíos que Gordon y Doyle (2015) presentan sobre la enseñanza de la responsabilidad personal y social y la transferencia del aprendizaje y las investigaciones sobre la validación del constructo "Responsabilidad social del estudiante universitario".

En 2015, Buyolo García señala que de las 71 universidades españolas que tienen implantada la titulación de ADE, tan solo 24 imparten alguna asignatura relacionada con la Responsabilidad Social Empresarial y de ese 32\%, solo la UFV establece una evaluación continua que incluye la interiorización de valores y aptitudes, porque como dice Echainiz Barrondo "Lo importante no es saber lo que está bien, sino hacer lo que está bien".

Otras investigaciones nos proporcionan modelos de análisis del impacto del uso de metodologías activas, como nuestras prácticas sociales, en el aprendizaje del estudiante (Robledo Ramón, Fidalgo Redondo, Arias Gundín y Álvarez Fernández,2015) o procesos para la validación de instrumentos de evaluación del trabajo cooperativo, que es una de los métodos que empleamos en la asignatura Responsabilidad social en la Universidad Francisco de Vitoria (García Cabrera, González López y Mérida Serrano, 2012).

\section{Objeto de estudio}

Con la asignatura de Responsabilidad Social no pretendemos enseñar el valor del compromiso social, sino ayudar al universitario "a descubrirlo por sí mismo" (López Quintás, 2003), porque el valor de la RSEU no se impone de forma coactiva, desde fuera, como algo extraño y ajeno.

Para ello, nos planteamos esta materia universitaria desde una perspectiva amplia que integra tres ámbitos de realización diferentes y complementarios: aula, prácticas sociales y acompañamiento, con el fin de que el alumno conecte la teoría del aula y la experiencia de las prácticas sociales, con su vida universitaria, con su día a día.

Las clases en el aula tienen como objetivo provocar en el alumno una reflexión sobre el sentido de la responsabilidad social desde el respeto a la dignidad humana.

El objetivo de las prácticas sociales es hacer la experiencia personal de que sí es posible hacer algo concreto para contribuir a la mejora de la sociedad. Para ello, se asigna a cada alumno un proyecto social en el que debe participar a lo largo de todo el curso en colaboración con una ONG. 
A través del acompañamiento personal buscamos iluminar la experiencia vivida en las prácticas para conectarla con su presente universitario y su futuro profesional.

Para medir el grado de transformación que experimenta el alumno tras cursar esta asignatura, elaboramos una escala de evaluación de la responsabilidad social.

El primer paso para la construcción de la escala de medida del grado de RSEU fue determinar los rasgos que definen esta variable. Para ello, aprovechamos la información obtenida a través del seguimiento personal de los alumnos que han cursado la asignatura durante cursos académicos anteriores.

Una vez definidas las dimensiones del concepto de RSEU, redactamos los ítems de la escala.

El instrumento de medición resultante (Apéndice) está estructurado sobre las cuatro dimensiones que conforman el constructo de RSEU (de la Calle, García Ramos y Giménez Armentía, 2007).

Compromiso con los demás y el entorno

Descubrimiento personal de los valores

Formación de la responsabilidad social

Planteamiento del ejercicio profesional desde el compromiso social

Ítem-criterio:

Valora globalmente el grado de responsabilidad social del estudiante universitario. Este ítem se introduce como variable criterio para ayudarnos a saber qué mide nuestro instrumento. Una buena medición del grado de compromiso y responsabilidad social del estudiante universitario se tiene que traducir en correlaciones altas y significativas entre cada uno de los ítems y el ítem criterio, así como entre el promedio de los ítems y el ítem criterio.

\section{Método}

\section{Objetivos}

El objetivo general de este estudio es evaluar el impacto de la enseñanza de la asignatura de RSEU sobre los alumnos de distintas titulaciones universitarias, mediante una herramienta fiable y válida.

Los objetivos específicos son:

Medir la existencia o no de impacto en las distintas titulaciones.

Encontrar posibles pautas comunes, entre titulaciones, en cuanto a los factores que configuran la RSEU.

\section{Hipótesis}

Se tienen tres hipótesis principales a contrastar en el estudio. Las tres están relacionadas con tres tipos de posibles impactos: (1) La asignatura tiene un impacto general sobre los alumnos, (2) Las diferentes dimensiones que componen la RSEU tienen impactos diferentes sobre los alumnos y (3) La asignatura tiene diferente impacto en los estudiantes de cada titulación. 


\section{Instrumento}

El instrumento que se propone para la medida está estructurado en cuatro dimensiones y 21 ítems como ya hemos descrito. Los estudios de fiabilidad y validez del instrumento utilizado muestran índices de consistencia interna más que aceptables o satisfactorios (entre .85 y .92, fiabilidad general) y los análisis factoriales exploratorios y confirmatorios indican la presencia de un constructo único, sobre la base de tres dimensiones correlacionadas, que reúne un $42.38 \%$ de la varianza. Como método de extracción de factores se aplica el procedimiento de componentes principales. La rotación factorial utilizada fue la oblicua Oblimin.

Los índices analizados (Chi-Cuadrado/gl, TLI , CFI y RMSEA) para evaluar el ajuste muestran que este es el modelo con mejor ajuste.

Un estudio detallado acerca de la fiabilidad y la validez de este instrumento se muestra en García, de la Calle, Valbuena y de Dios (2015).

\section{Población, muestra y procedimiento de recolección de datos}

Los estudiantes de $2^{\circ}$ de la UFV que cursan la asignatura de RSEU son la población objeto de estudio. El total de alumnos matriculados en esta asignatura en el curso 20122013 fue de 639. La selección de la muestra se realizó mediante un procedimiento de muestreo por cuotas, considerando la titulación como la característica fundamental, donde la muestra debería ser similar a la población objeto de estudio -estudiantes de $2^{\circ}$ de la UFV-.

La información se recoge mediante encuesta personal. El trabajo de campo se realizó en dos momentos: (1) en Octubre del 2012 se aplicó a los alumnos cuando la docencia de las asignaturas apenas había comenzado (pretest) y (2) en Mayo del 2013 se aplicó de nuevo a los alumnos cuando ya habían cursado completamente la asignatura (postest). Las muestras del pretest y el postest son independientes, ya que los cuestionarios son anónimos y no se identifica al individuo que había contestado el pretest y postest, para poder así relacionar ambas muestras. La Tabla 1 recoge la ficha técnica del muestreo.

Tabla 1

Ficha técnica de la investigación

\begin{tabular}{ll}
\hline Características & Encuesta \\
\hline Universo & $\begin{array}{l}\text { Alumnos de } 2^{\circ} \text { de la UFV que cursen la asignatura de Responsa- } \\
\text { bilidad Social }\end{array}$ \\
Procedimiento de muestreo & $\begin{array}{l}\text { Por cuotas. Estratificación según titulación } \\
\text { Recogida de información }\end{array}$ \\
Encuesta personal a los alumnos en clase \\
Tamaño de la muestra & 404 (Pretest) / 353 (Postest) encuestas recogidas \\
Error muestral & $\pm 3 \%$ \\
Nivel de confianza & $95 \%$ para el caso más desfavorable $\mathrm{p}=\mathrm{q}=0,5$ \\
Trabajo de Campo & Noviembre 2012 (Pretest) y Mayo 2013(Postest) \\
\hline
\end{tabular}


Finalmente, se obtuvieron un total de 757 encuestas: 404 encuestas en el pretest (63.2\% de la población) y 353 encuestas en el postest (55.2\% de la población) -, identificándose a posteriori el siguiente perfil de la muestra (Tabla 2).

Tabla 2

Perfil de la muestra

\begin{tabular}{lllll}
\hline & \multicolumn{3}{l}{ Pretest } & \multicolumn{2}{l}{ Postest } \\
\cline { 2 - 6 } Titulaciones & $\mathrm{N}$ & $\%$ & $\mathrm{~N}$ & $\%$ \\
\hline Administración y Dirección de Empresas (ADE) & 31 & 7.67 & 24 & 6.80 \\
Biotecnología (BIO) & 41 & 10.15 & 41 & 11.61 \\
Ciencias de la Actividad Física y del Deporte (CAFyD) & 63 & 15.59 & 46 & 13.03 \\
Comunicación Audiovisual (COM) & 37 & 9.16 & 49 & 13.88 \\
Derecho (DER) & 20 & 4.95 & 20 & 5.67 \\
Diseño y Bellas Artes (DIS + BBAA) & 39 & 9.65 & 29 & 8.22 \\
Educación Infantil + Educación Primaria (EDU) & 41 & 10.15 & 21 & 5.95 \\
ADE+ Derecho (Excellens) (EXC) & 9 & 2.23 & 10 & 2.83 \\
Fisioterapia (FIS) & 38 & 9.41 & 37 & 10.48 \\
Informática (INF) & 15 & 3.71 & 15 & 4.25 \\
Marketing (MAR) & 10 & 2.48 & 11 & 3.12 \\
Periodismo (PER) & 49 & 12.13 & 34 & 9.63 \\
Publicidad (PUB) & 11 & 2.72 & 16 & 4.53 \\
\hline
\end{tabular}

\section{Procedimiento de análisis de datos}

Para la codificación y análisis de los datos se emplea el programa estadístico SPSS versión 21 (SPSS Inc., Chicago, USA). Se realizan análisis descriptivos (medidas de tendencia central y desviación típica) y con el fin de contrastar las diferencias significativas a nivel estadístico entre ambos momentos (antes de cursar la asignatura y después de haberla cursado) se realizan análisis de diferencias mediante pruebas paramétricas t-student para muestras independientes y Análisis de Varianza (ANOVA) de un factor y de dos factores.. Se verifican los supuestos de independencia de los datos, normalidad y homocedasticidad. Se realiza un contraste t-Student, sin distinguir entre titulaciones, sobre una dimensión agregada (suma de las cuatro dimensiones). Se realizan Análisis de Varianza (ANOVA) de un factor, sobre cada una de las cuatro dimensiones y sobre el ítem criterio. Para estas seis variables cuantitativas, se realiza ANOVA de dos factores para evaluar los efectos de las dos posibles fuentes de variación, así como la interacción entre ambas: (a) pertenencia al grupo pretest o postest y (b) pertenencia a una titulación determinada. Se realiza, también, ANOVA de un factor sobre las cuatro dimensiones y el ítem criterio, para cada una de las titulaciones. Prácticamente, en todos los análisis se ha empleado un nivel de confianza del $95 \%$ o superior. 


\section{Resultados}

\section{Estadísticos descriptivos}

En la Tablas 3 y 4 se presentan los estadísticos descriptivos de las dimensiones en el pretest y postest para las distintas titulaciones. Las puntuaciones de cada dimensión se calculan como sumatorio de los ítems que la componen.

Todas las titulaciones tienen un grado de compromiso social medio en todas las dimensiones, tanto en el pretest como en el postest por encima de 4.1, salvo algunas excepciones.

En general, en todas las titulaciones, la satisfacción media en cada dimensión, obtenida en el postest es más elevada que en pretest, salvo algunas excepciones mínimas.

Por tanto, los resultados descriptivos parecen indicar posibles diferencias de medias entre el pretest y el postest en casi todas las titulaciones. Estos resultados preliminares requieren la utilización de métodos inferenciales de tratamiento de datos que nos permitan concluir si las diferencias son significativas o no.

Tabla 3

Estadísticos descriptivos de las dimensiones por titulaciones (pretest (PR) y postest (PO))

\begin{tabular}{|c|c|c|c|c|c|c|c|c|c|c|c|c|c|}
\hline \multicolumn{14}{|c|}{ TITULACIÓN } \\
\hline & & $\begin{array}{l}\mathrm{ADE} \\
\text { (PR) }\end{array}$ & $\begin{array}{l}\mathrm{ADE} \\
(\mathrm{PO}) \\
\end{array}$ & $\begin{array}{l}\mathrm{BIO} \\
\text { (PR) } \\
\end{array}$ & $\begin{array}{l}\mathrm{BIO} \\
(\mathrm{PO}) \\
\end{array}$ & $\begin{array}{l}\text { CAFyD } \\
\text { (PR) }\end{array}$ & $\begin{array}{l}\text { CAFyD } \\
(\mathrm{PO})\end{array}$ & $\begin{array}{c}\mathrm{COM} \\
(\mathrm{PR})\end{array}$ & $\begin{array}{l}\mathrm{COM} \\
(\mathrm{PO})\end{array}$ & $\begin{array}{l}\text { DER } \\
\text { (PR) } \\
\end{array}$ & $\begin{array}{l}\mathrm{DER} \\
(\mathrm{PO}) \\
\end{array}$ & $\begin{array}{c}\text { DIS+BBAA } \\
(\mathrm{PR})\end{array}$ & $\begin{array}{c}\text { DIS+BBAA } \\
(\mathrm{PO})\end{array}$ \\
\hline & $\mathrm{N}$ & 31 & 24 & 41 & 41 & 63 & 46 & 37 & 49 & 20 & 20 & 39 & 29 \\
\hline \multirow{4}{*}{$\sum_{\bar{\sigma}}^{\rightleftarrows}$} & $\bar{X}$ & 4.3 & 4.4 & 4.4 & 4.7 & 4.1 & 4.6 & 4.1 & 4.7 & 3.9 & 5.3 & 4.4 & 4.8 \\
\hline & $S$ & 0.71 & 0.72 & 0.77 & 0.77 & 0.86 & 0.61 & 0.65 & 0.60 & 0.70 & 0.55 & 0.66 & 0.60 \\
\hline & Mo & 3.8 & 4.0 & 4.2 & 4.6 & 4.4 & 4.4 & 4.0 & 4.6 & 4.0 & 5.4 & 4.6 & 5.2 \\
\hline & $\mathrm{Md}$ & 4.4 & 4.5 & 4.4 & 4.6 & 4.2 & 4.6 & 4.2 & 4.8 & 4.0 & 5.4 & 4.6 & 4.8 \\
\hline \multirow{4}{*}{$\sum_{0}^{N}$} & $\bar{X}$ & 4.5 & 4.6 & 4.8 & 5.1 & 4.5 & 4.9 & 4.6 & 5.0 & 4.4 & 5.5 & 4.8 & 5.2 \\
\hline & $S$ & 0.72 & 0.85 & 0.81 & 0.78 & 0.77 & 0.71 & 0.67 & 0.65 & 0.80 & 0.42 & 0.70 & 0.66 \\
\hline & Mo & 4.2 & 5.6 & 5.6 & 5.4 & 4.6 & 4.2 & 4.6 & 4.8 & 4.0 & 5.8 & 5.2 & 5.0 \\
\hline & $\mathrm{Md}$ & 4.6 & 4.7 & 5.0 & 5.2 & 4.6 & 4.8 & 4.6 & 5.0 & 4.4 & 5.6 & 4.6 & 5.2 \\
\hline \multirow{4}{*}{$\sum_{\overline{0}}^{\infty}$} & $\bar{X}$ & 4.1 & 4.5 & 4.2 & 4.5 & 4.1 & 4.5 & 3.9 & 4.3 & 4.1 & 5.4 & 4.0 & 4.2 \\
\hline & S & 0.98 & 0.75 & 1.11 & 1.14 & 0.99 & 0.84 & 0.92 & 0.83 & 0.73 & 0.39 & 0.91 & 0.73 \\
\hline & Mo & 3.2 & 4.6 & 4.4 & 4.8 & 4.6 & 4.8 & 3.8 & 4.8 & 3.0 & 5.6 & 3.2 & 4.4 \\
\hline & $\mathrm{Md}$ & 4.0 & 4.6 & 4.4 & 4.8 & 4.2 & 4.5 & 4.0 & 4.4 & 4.1 & 5.5 & 4.2 & 4.4 \\
\hline \multirow{4}{*}{$\sum_{0}^{+}$} & $\bar{X}$ & 4.4 & 4.6 & 4.6 & 5.0 & 4.5 & 4.8 & 4.5 & 4.7 & 4.8 & 5.5 & 4.8 & 4.8 \\
\hline & $S$ & 0.86 & 0.96 & 1.03 & 0.71 & 0.92 & 0.69 & 0.68 & 0.66 & 0.65 & 0.34 & 0.81 & 0.73 \\
\hline & Mo & 3.8 & 3.6 & 4.6 & 4.6 & 4.8 & 4.4 & 4.4 & 4.4 & 5.4 & 5.2 & 5.6 & 5.0 \\
\hline & $\mathrm{Md}$ & 4.4 & 4.4 & 4.8 & 5.0 & 4.6 & 4.8 & 4.4 & 4.8 & 4.8 & 5.5 & 4.8 & 5.0 \\
\hline
\end{tabular}


Tabla 4

Estadísticos descriptivos de las dimensiones por titulaciones (pretest (PR) y postest (PO))

\begin{tabular}{|c|c|c|c|c|c|c|c|c|c|c|c|c|c|c|c|}
\hline \multicolumn{16}{|c|}{ TITULACIÓN } \\
\hline & & $\begin{array}{l}\text { EDU } \\
\text { (PR) }\end{array}$ & $\begin{array}{l}\text { EDU } \\
\text { (PO) }\end{array}$ & $\begin{array}{l}\text { EXC } \\
\text { (PR) }\end{array}$ & $\begin{array}{l}\text { EXC } \\
\text { (PO) }\end{array}$ & $\begin{array}{c}\text { FIS } \\
\text { (PR) }\end{array}$ & $\begin{array}{c}\text { FIS } \\
\text { (PO) }\end{array}$ & $\begin{array}{l}\text { INF } \\
\text { (PR) }\end{array}$ & $\begin{array}{l}\text { INF } \\
\text { (PO) }\end{array}$ & $\begin{array}{l}\text { MAR } \\
\text { (PR) }\end{array}$ & $\begin{array}{c}\text { MAR } \\
\text { (PO) }\end{array}$ & $\begin{array}{l}\text { PER } \\
\text { (PR) }\end{array}$ & $\begin{array}{l}\text { PER } \\
\text { (PO) }\end{array}$ & $\begin{array}{l}\text { PUB } \\
\text { (PR) }\end{array}$ & $\begin{array}{l}\text { PUB } \\
\text { (PO) }\end{array}$ \\
\hline & $\mathrm{N}$ & 41 & 21 & 9 & 10 & 38 & 37 & 15 & 15 & 10 & 11 & 49 & 34 & 16 & 11 \\
\hline \multirow{4}{*}{$\sum_{\bar{\sigma}}^{\bar{B}}$} & $\bar{X}$ & 4.9 & 5.3 & 4.6 & 4.8 & 4.3 & 4.8 & 3.3 & 4.1 & 4.5 & 5.3 & 4.5 & 4.8 & 5.0 & 3.9 \\
\hline & S & 0.65 & 0.47 & 0.43 & 1.03 & 0.87 & 1.04 & 1.27 & 1.04 & 0.99 & 0.48 & 0.86 & 0.81 & 0.93 & 0.52 \\
\hline & Mo & 5.0 & 5.2 & 4.2 & 5.0 & 3.6 & 4.6 & 2.6 & 4.8 & 4.8 & 5.4 & 4.8 & 4.6 & 4.6 & 3.6 \\
\hline & $\mathrm{Md}$ & 5.0 & 5.2 & 4.6 & 5.1 & 4.4 & 5.0 & 3.2 & 4.6 & 4.8 & 5.4 & 4.6 & 5.0 & 4.9 & 3.6 \\
\hline \multirow{4}{*}{ 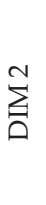 } & $\bar{X}$ & 5.2 & 5.6 & 5.2 & 5.1 & 4.9 & 5.0 & 3.6 & 4.2 & 4.9 & 5.4 & 4.9 & 5.1 & 5.5 & 4.7 \\
\hline & $S$ & 0.57 & 0.36 & 0.61 & 0.95 & 0.85 & 1.04 & 1.17 & 0.97 & 0.82 & 0.37 & 0.85 & 0.73 & 0.77 & 0.47 \\
\hline & Mo & 4.6 & 5.6 & 4.6 & 6.0 & 5.4 & 6.0 & 2.6 & 4.4 & 3.6 & 5.4 & 5.8 & 6.0 & 5.6 & 3.8 \\
\hline & $\mathrm{Md}$ & 5.2 & 5.6 & 5.4 & 5.3 & 5.0 & 5.2 & 3.6 & 4.4 & 5.3 & 5.4 & 5.0 & 5.2 & 5.6 & 4.6 \\
\hline \multirow{4}{*}{$\sum_{0}^{\infty}$} & $\bar{X}$ & 4.6 & 5.2 & 4.6 & 4.7 & 4.0 & 4.7 & 3.1 & 3.8 & 4.3 & 5.2 & 4.2 & 4.6 & 4.9 & 3.4 \\
\hline & $S$ & 1.03 & 0.52 & 0.54 & 1.00 & 0.99 & 1.04 & 1.28 & 1.21 & 0.84 & 0.46 & 0.93 & 1.05 & 1.16 & 1.01 \\
\hline & Mo & 5.0 & 4.6 & 4.4 & 4.6 & 3.0 & 4.8 & 4.0 & 4.4 & 4.2 & 5.4 & 4.0 & 4.6 & 5.4 & 2.8 \\
\hline & $\mathrm{Md}$ & 4.8 & 5.2 & 4.8 & 4.8 & 4.1 & 4.8 & 2.8 & 4.2 & 4.2 & 5.2 & 4.2 & 4.6 & 5.4 & 3.0 \\
\hline \multirow{4}{*}{$\sum_{\overline{0}}^{+}$} & $\bar{X}$ & 5.0 & 5.5 & 5.0 & 5.0 & 5.0 & 5.0 & 3.7 & 4.3 & 4.7 & 5.3 & 5.1 & 5.0 & 5.2 & 3.9 \\
\hline & $S$ & 0.76 & 0.34 & 0.68 & 1.21 & 0.77 & 1.06 & 1.17 & 1.10 & 0.99 & 0.42 & 0.70 & 0.89 & 0.89 & 0.65 \\
\hline & Mo & 6.0 & 5.6 & 4.4 & 6.0 & 4.8 & 6.0 & 2.4 & 4.2 & 4.8 & 5.2 & 4.8 & 6.0 & 5.6 & 3.2 \\
\hline & $\mathrm{Md}$ & 5.2 & 5.6 & 5.2 & 5.5 & 5.0 & 5.2 & 3.4 & 4.4 & 4.8 & 5.2 & 5.2 & 5.2 & 5.4 & 3.6 \\
\hline
\end{tabular}

\section{Contrastes sobre diferencias de medias}

Se contrastan las hipótesis de ausencia de impacto a nivel general, por dimensión y por titulación a partir de contrastes de diferencias de medias (hipótesis nulas).

\section{Contraste de diferencia de medias para la ausencia de impacto general}

Para contrastar la hipótesis de ausencia de impacto general entre el pretest y postest, se calcula la dimensión agregada, como sumatorio de las cuatro dimensiones y se realiza un contraste $t$ de Student. El resultado del contraste indica que la diferencia es significativa a favor del postest. Véase Tabla 5.

También se lleva a cabo un modelo factorial de análisis de varianza. Este modelo sirve para evaluar el efecto individual y conjunto de dos o más factores (titulaciones y grupo) sobre una variable dependiente cuantitativa (ítem 21 o dimensión).

En nuestro caso permite determinar si la interacción entre los factores grupo (pretest/ postest) y titulación afecta a la variable dependiente. 
Los p-valores menores de 0.05 indican que los grupos definidos por pretest y postest poseen resultados medios significativamente diferentes y que los resultados medios entre titulaciones son significativamente diferentes también. La información sobre el efecto de la interacción grupo*titulación, indica que las diferencias de resultados con la impartición de la asignatura de Responsabilidad Social que se dan entre el pretest y el postest, a favor del postest, no son las mismas entre titulaciones, o dicho de otra forma, las diferencias entre titulaciones no son las mismas en los dos grupos (pretest y postest). Véase Tabla 6.

Tabla 5

Resultados en la Dimensión Agregada (Contraste t-Student)

\begin{tabular}{|c|c|c|c|c|c|c|c|}
\hline & \multirow[t]{2}{*}{$\mathrm{F}$} & \multicolumn{2}{|c|}{$\begin{array}{c}\text { Prueba de } \\
\text { Levene para } \\
\text { la igualdad } \\
\text { de varianzas }\end{array}$} & \multicolumn{3}{|c|}{$\begin{array}{c}\text { Prueba T para la igualdad de } \\
\text { medias }\end{array}$} & \multirow[t]{2}{*}{$\begin{array}{l}\text { Dirección } \\
\text { Significativa }\end{array}$} \\
\hline & & Sig. & $\mathrm{t}$ & gl & $\begin{array}{l}\text { Diferencia } \\
\text { de medias }\end{array}$ & & \\
\hline \multirow{2}{*}{$\begin{array}{l}\text { DIM } \\
\text { TOT }\end{array}$} & $\begin{array}{l}\text { varianzas } \\
\text { iguales }\end{array}$ & .166 & .683 & -7.111 & 717 & $-8.00842^{* * *}$ & A favor del postest \\
\hline & $\begin{array}{c}\text { No varianzas } \\
\text { iguales }\end{array}$ & 0.0 .1 & 0.0 .2 & -7.114 & 711.224 & -8.00842 & \\
\hline
\end{tabular}

*** Diferencia significativa al 1\% $(\mathrm{p}<.01)$

Tabla 6

Resultados para la Dimensión Agregada (ANOVA de dos factores)

\begin{tabular}{lccccc}
\hline Origen & $\begin{array}{c}\text { Suma de } \\
\text { cuadrados } \\
\text { tipo III }\end{array}$ & gl & $\begin{array}{c}\text { Media } \\
\text { cuadrática }\end{array}$ & F & Dirección Significativa \\
\hline GRUPO & 10160.547 & 1 & 10160.547 & 46.732 & A favor del postest $^{* * *}$ \\
\hline TITULACIÓN & 13064.114 & 10 & 1306.411 & 6.009 & A favor del postest*** $^{*}$ \\
\hline GRUPO * TITULACIÓN & 4146.474 & 10 & 414.647 & 1.907 & A favor del postest $^{* *}$ \\
\hline
\end{tabular}

a. $\mathrm{R}$ cuadrado $=.186$ ( $\mathrm{R}$ cua-

drado corregida $=, 156)$

** Diferencia significativa al 5\% $(\mathrm{p}<.05)$

*** Diferencia significativa al 1\% $(\mathrm{p}<.01)$

\section{Contraste de diferencia de medias para la ausencia de impacto por dimensión}

Se realizan Análisis de Varianza de un Factor con el ítem criterio (ítem 21) y con las Dimensiones 1, 2, 3 y 4. Los resultados indican que las diferencias son significativas a favor del postest. Véase la Tabla 7. 
Los Análisis de Varianza de dos factores revelan diferencias significativas en todas las dimensiones y en el ítem criterio a favor del postest, así como diferencias significativas entre titulaciones. Los efectos cruzados entre titulación y grupo (pretest/postet) son significativos. La única dimensión que presenta una interacción entre titulaciones y pretest y postest no significativa es la Dimensión 2. Las Tablas 8, 9, 10, 11 y 12 muestran estos resultados.

Tabla 7

Resultados para el item criterio y para las dimensiones (ANOVA de un factor)

\begin{tabular}{|c|c|c|c|c|c|c|}
\hline & & $\begin{array}{l}\text { Suma de } \\
\text { cuadrados }\end{array}$ & $\mathrm{gl}$ & $\begin{array}{c}\text { Media } \\
\text { cuadrática }\end{array}$ & $\mathrm{F}$ & Dirección Significativa \\
\hline \multirow{3}{*}{$\begin{array}{c}\text { ITEM } \\
21 .\end{array}$} & Inter-grupos & 59.332 & 1 & 59.332 & 53.005 & \multirow{3}{*}{ A favor del postest ${ }^{* * *}$} \\
\hline & Intra-grupos & 838.415 & 749 & 1.119 & & \\
\hline & Total & 897.747 & 750 & & & \\
\hline \multirow{3}{*}{ DIM 1.} & Inter-grupos & 1139.147 & 1 & 1139.147 & 69.129 & \multirow{3}{*}{ A favor del postest ${ }^{* * *}$} \\
\hline & Intra-grupos & 12326.001 & 748 & 16.479 & & \\
\hline & Total & 13465.148 & 749 & & & \\
\hline \multirow{3}{*}{ DIM 2.} & Inter-grupos & 568.687 & 1 & 568.687 & 34.964 & \multirow{3}{*}{ A favor del postest ${ }^{* * *}$} \\
\hline & Intra-grupos & 12019.931 & 739 & 16.265 & & \\
\hline & Total & 12588.618 & 740 & & & \\
\hline \multirow{3}{*}{ DIM 3.} & Inter-grupos & 1095.207 & 1 & 1095.207 & 45.159 & \multirow{3}{*}{ A favor del postest ${ }^{* * *}$} \\
\hline & Intra-grupos & 18068.073 & 745 & 24.252 & & \\
\hline & Total & 19163.280 & 746 & & & \\
\hline \multirow{3}{*}{ DIM 4.} & Inter-grupos & 352.284 & 1 & 352.284 & 19.081 & \multirow{3}{*}{ A favor del postest ${ }^{* * *}$} \\
\hline & Intra-grupos & 13754.415 & 745 & 18.462 & & \\
\hline & Total & 14106.699 & 746 & & & \\
\hline
\end{tabular}

*** Diferencia significativa al 1\% $(\mathrm{p}<.01)$

Tabla 8

Resultados para el Ítem Criterio (ANOVA de dos factores)

\begin{tabular}{lccccc}
\hline & $\begin{array}{c}\text { Suma de } \\
\text { cuadrados tipo III }\end{array}$ & gl & $\begin{array}{c}\text { Media } \\
\text { cuadrática }\end{array}$ & F & $\begin{array}{c}\text { Dirección } \\
\text { Significativa }\end{array}$ \\
\hline TITULACIÓN & 35.144 & 10 & 3.514 & 3.209 & A favor del postest $^{* * *}$ \\
\hline GRUPO & 59.409 & 1 & 59.409 & 54.243 & A favor del postest $^{* * *}$ \\
\hline TITULACIÓN * GRUPO & 22.579 & 10 & 2.258 & 2.062 & A favor del postest $^{* *}$ \\
\hline
\end{tabular}

a. $\mathrm{R}$ cuadrado $=.158(\mathrm{R}$

cuadrado corregida $=.128$ )

** Diferencia significativa al 5\% $(\mathrm{p}<.05)$

*** Diferencia significativa al 1\% $(\mathrm{p}<.01)$ 
Tabla 9

Resultados para la Dimensión 1 (ANOVA de dos factores)

\begin{tabular}{lccccc}
\hline & $\begin{array}{c}\text { Suma de } \\
\text { cuadrados tipo III }\end{array}$ & gl & $\begin{array}{c}\text { Media } \\
\text { cuadrática }\end{array}$ & F & Dirección Significativa \\
\hline TITULACIÓN & 716.675 & 10 & 71.668 & 4.542 & A favor del postest*** $^{* * 5}$ \\
\hline GRUPO & 1031.545 & 1 & 1031.545 & 65.374 & A favor del postest*** $^{*}$ \\
\hline $\begin{array}{l}\text { TITULACIÓN * } \\
\text { GRUPO }\end{array}$ & 399.861 & 10 & 39.986 & 2.534 & A favor del postest** $^{*}$ \\
\hline
\end{tabular}

a. $\mathrm{R}$ cuadrado $=, 193(\mathrm{R}$ cuadrado corregida $=, 165$

*** Diferencia significativa al $1 \%(\mathrm{p}<.01)$

Tabla 10

Resultados para la Dimensión 2 (ANOVA de dos factores)

\begin{tabular}{lccccc}
\hline & $\begin{array}{c}\text { Suma de } \\
\text { cuadrados tipo III }\end{array}$ & gl & $\begin{array}{c}\text { Media } \\
\text { cuadrática }\end{array}$ & F & Dirección Significativa \\
\hline TITULACIÓN & 1022.919 & 10 & 102.292 & 6.608 & A favor del postest** $^{* *}$ \\
\hline GRUPO & 429.853 & 1 & 429.853 & 27.767 & A favor del postest** $^{* * 3}$ \\
\hline $\begin{array}{l}\text { TITULACIÓN } \\
\text { GRUPO }\end{array}$ & 224.897 & 10 & 22.490 & 1.453 & \\
\hline
\end{tabular}

a. $\mathrm{R}$ cuadrado $=.165(\mathrm{R}$ cuadrado corregida $=.135)$

*** Diferencia significativa al $1 \%(\mathrm{p}<.01)$

Tabla 11

Resultados para la Dimensión 3 (ANOVA de dos factores)

\begin{tabular}{lccccc}
\hline & $\begin{array}{c}\text { Suma de } \\
\text { cuadrados tipo III }\end{array}$ & gl & $\begin{array}{c}\text { Media } \\
\text { cuadrática }\end{array}$ & F & Dirección Significativa \\
\hline TITULACIÓN & 1071.702 & 10 & 107.170 & 4.639 & A favor del postest ${ }^{* * *}$ \\
\hline GRUPO & 1028.217 & 1 & 1028.217 & 44.505 & A favor del postest*** \\
\hline $\begin{array}{l}\text { TITULACIÓN } \\
\text { GRUPO }\end{array}$ & 379.556 & 10 & 37.956 & 1.643 & A favor del postest \\
\hline
\end{tabular}

a. $\mathrm{R}$ cuadrado $=.156(\mathrm{R}$ cuadrado corregida $=.127)$

${ }^{*}$ Diferencia significativa al 10\% $(\mathrm{p}<.10)$

*** Diferencia significativa al 1\% $(\mathrm{p}<.01)$ 
Tabla 12

Resultados para la Dimensión 4 (ANOVA de dos factores)

\begin{tabular}{lccccc}
\hline & $\begin{array}{c}\text { Suma de } \\
\text { cuadrados tipo III }\end{array}$ & gl & $\begin{array}{c}\text { Media } \\
\text { cuadrática }\end{array}$ & F & Dirección Significativa \\
\hline TITULACIÓN & 1117.229 & 10 & 111.723 & 6.454 & A favor del postest $^{* * *}$ \\
\hline GRUPO & 428.835 & 1 & 428.835 & 24.773 & A favor del postest $^{* * *}$ \\
\hline $\begin{array}{l}\text { TITULACIÓN } \\
\text { GRUPO }\end{array}$ & 342.801 & 10 & 34.280 & 1.980 & A favor del postest $^{* *}$ \\
\hline
\end{tabular}

a. $\mathrm{R}$ cuadrado $=.149(\mathrm{R}$ cuadrado corregida $=.120)$

${ }^{* *}$ Diferencia significativa al 5\% ( $\left.<<.05\right)$

*** Diferencia significativa al $1 \%(\mathrm{p}<.01)$

\section{Contraste de diferencia de medias para la ausencia de impacto por titulaciones}

Se realizan contrastes t-Student para la diferencia de medias entre el pretest y postest (muestras independientes). Previamente, se realiza el test de Levene de Homogeneidad de Varianzas.

En la Tabla 13 se presentan los resultados obtenidos en el análisis de significatividad de la diferencia de medias por titulaciones.

Tabla 13

Resumen diferencias significativas en cada dimensión por titulaciones

\begin{tabular}{|c|c|c|c|c|c|}
\hline TITULACIÓN & $\begin{array}{c}\text { ÍTEM } \\
\text { CRITERIO }\end{array}$ & DIM 1 & DIM 2 & DIM 3 & DIM4 \\
\hline $\mathrm{ADE}$ & $* *$ & & & * & \\
\hline $\mathrm{BIO}$ & & * & & & $* *$ \\
\hline CAFyD & $* * *$ & $* * *$ & * & * & * \\
\hline $\mathrm{COM}$ & $* *$ & $* * *$ & $* *$ & * & \\
\hline DER & $* * *$ & $* * *$ & $* * *$ & $* * *$ & $* * *$ \\
\hline BBAA+DIS & * & $* *$ & $* *$ & & \\
\hline EDU & & $* * *$ & $* * *$ & $* * *$ & $* * *$ \\
\hline \multicolumn{6}{|c|}{ ADE+DER (EXC) } \\
\hline FIS & * & $* *$ & & $* *$ & \\
\hline INF & & * & & & \\
\hline MAR & & $* *$ & & $* *$ & \\
\hline PER & & * & & & \\
\hline PUB & $* * *$ & $* * *$ & $* *$ & $* * *$ & $* * *$ \\
\hline
\end{tabular}

${ }^{*}$ Diferencias significativas al nivel de significación .10

** Diferencias significativas al nivel de significación .05

** *Diferencias significativas al nivel de significación .01 
A un nivel de significación del 10\%, en ADE+Derecho (Excellens) no parece haber ningún impacto de la asignatura de RSEU. En los alumnos de Ingeniería Informática y Periodismo parece haber un impacto significativo de la docencia de la asignatura de RSEU a un nivel de $10 \%$. En las titulaciones de Publicidad, CAFyD y Derecho parece haber impacto en los alumnos, en todas las dimensiones y en el Ítem criterio.

Las Dimensiones 1 (Compromiso con los demás y el entorno) y 3 (Formación de la responsabilidad social) son las que más impacto parecen tener entre los alumnos, principalmente la Dimensión 1. La Dimensión 4 (Planteamiento del ejercicio profesional desde el compromiso social) es la dimensión que menos impacto suele tener entre los alumnos.

La asignatura de Responsabilidad Social parece tener impacto sobre el compromiso de los alumnos con los demás y su entorno (DIM 1), salvo en las titulaciones de ADE y el doble grado de ADE+DER (EXC). El descubrimiento de los valores (DIM 2) parece verse reforzado después de cursar la asignatura en aquellas titulaciones relacionadas con la creatividad como son Comunicación Audiovisual, Publicidad, Bellas Artes y Diseño y Educación Infantil y Primaria. También parece que hay un impacto en titulaciones como el Derecho y Ciencias de la Actividad Física y Deporte, donde los valores tienen un papel muy importante en el ejercicio de la profesión. Esta hipótesis se ve afianzada en el impacto que tiene para los estudiantes de estas titulaciones el planteamiento de su ejercicio profesional desde su compromiso social (DIM 4). Este impacto también se presenta en los biotecnólogos, los maestros y los publicistas.

Los estudiantes de Administración de Empresas, de Comunicación Audiovisual, de Fisioterapia y de Bellas Artes y Diseño, a pesar de que la experiencia de cursar la asignatura parece no tener un impacto en todas las dimensiones, sí parecen haber impacto a nivel general en su capacidad de compromiso y responsabilidad social. Sin embargo, los estudiantes de magisterio, si bien experimentan una transformación en su compromiso, en el descubrimiento de los valores (DIM 2), en su formación en responsabilidad social (DIM 3) y en el planteamiento de su ejercicio profesional desde su compromiso social (DIM 4), no parecen experimentar una transformación, a nivel general, en su compromiso y su responsabilidad social. Esto podría explicarse como que los alumnos son capaces de identificar cada núcleo o dimensión por separado, pero a nivel global consideran que su compromiso y responsabilidad no ha cambiado de forma relevante.

\section{Discusión de resultados y conclusiones}

La asignatura de Responsabilidad Social del Universitario que se imparte en la Universidad parece tener un impacto general positivo sobre los estudiantes. Los resultados muestran diferencias significativas en la dimensión agregada (general) formada por el compromiso con los demás y el entorno, el descubrimiento personal de los valores, la formación en responsabilidad social y el planteamiento del ejercicio profesional desde el compromiso social. Esto muestra el impacto en la capacidad de compromiso y en la responsabilidad social del estudiante, después de pasar por la experiencia de cursar la asignatura. Parece también clave que este impacto es diferente entre titulaciones.

El análisis por dimensiones indica que cada una de las dimensiones, por separado, tiene impacto sobre los estudiantes. El compromiso de los estudiantes, el descubri- 
miento de los valores, su formación en responsabilidad social y el planteamiento de su profesión desde el compromiso social se ven reforzados, después de haber cursado la asignatura. También, en términos generales, su compromiso y responsabilidad social se ven mejorados después de haber cursado la asignatura. También hay un impacto diferente entre titulaciones, después de cursar la asignatura, excepto en el descubrimiento personal de los valores, donde no se encuentran diferencias significativas en la interacción entre el pretest y postest y las titulaciones.

El impacto por titulaciones de la asignatura revela que, efectivamente, los resultados son diferentes por titulaciones.

En la única titulación en la que no parece tener impacto la docencia de la asignatura de RSEU es en ADE+Derecho (Excellens). En las titulaciones de Periodismo, Derecho y CAFyD la docencia de la asignatura impacta positivamente en el compromiso con los demás y el entorno, valores, responsabilidad social, planteamiento profesional y en su compromiso social global.

La docencia de la asignatura parece tener un efecto positivo, prácticamente en todas las titulaciones (salvo en ADE y ADE+Derecho), en el grado de compromiso con los demás y el entorno. Sin embargo, el planteamiento profesional desde el compromiso social parece verse afectado positivamente, tras la docencia de la asignatura, únicamente en Biotecnología, CAFyD, Derecho, Educación y Publicidad.

En resumen, los resultados indican que la experiencia de cursar la asignatura de Responsabilidad Social, tiene impacto significativo en los estudiantes de la UFV, a nivel general, por dimensiones y titulaciones. Y que este impacto es diferente entre las titulaciones.

\section{Referencias bibliográficas}

Astin, A.J., Sax, L., \& Avalos, J. (1999). Long term effects of volunteerism during the undergraduate years. The Review Of Higher Education, 22(2), 187-202.

Buyolo García, F. (2015). Humanizar la empresa. Hacia una formación empresarial ética. Bubok Publishing S.L.

de la Calle Maldonado, C., García Ramos, J.M., \& Giménez Armentía, P. (2007). La formación de la responsabilidad social en la Universidad. Revista Complutense de Educación, 18(2), 47-66.

de la Calle Maldonado, C., García Ramos, J.M., Giménez Armentía, P., \& Ortega de la Fuente, M. (2008). Validación y medida de la responsabilidad social en la Universidad. Revista Complutense de Educación, 19(2) 358-404.

de Dios-Alija, T. (2014). Responsabilidad de la persona y sostenibilidad de las organizaciones. Madrid: Universidad Francisco de Vitoria.

Galán, J.I., Sáenz de Miera, A. (Eds.), \& de la Calle Maldonado, C. (Coord.) (2012). Reflexiones sobre la responsabilidad social corporativa en el siglo XXI. Salamanca: Ediciones Universidad de Salamanca.

García Cabrera, M., M. González López, I., \& Mérida Serrano, R. (2012). Validación del cuestionario de evaluación ACOES. Análisis del trabajo cooperativo en Educación Superior. Revista de Investigación Educativa, 30(1) 89-107. doi: 10.6018/rie.30.1.114091 
García Ramos, J.M., de la Calle Maldonado, C., Valbuena Martínez, M.C., \& de DiosAlija, T. (2015). Hacia la validación del constructo Responsabilidad Social del Estudiante Universitario (RSEU). Bordon, 68(1) 153-170.

Gordon, B. \& Doyle, S. (2015). Teaching personal and social responsibility and transfer of learning: opportunities and challenges for teachers and coaches. Journal of Teaching in Physical Education, 34, 152-161. doi:10.1123/jtpe.2013-0184.

Hernandez De Hahn, L. (2014). Socially responsible citizens: promoting gifts and talents that support social and humanitarian advancement. International Studies in Sociology of Education, 24(4), 415-434. doi: 10.1080/09620214.2014.979595

Lappalainen, P. (2011). Development Cooperation as Methodology for Teaching Social Responsibility to Engineers. European Journal of Engineering Education, 36(6), 513-519. doi:10.1080103043797.2011.619648

Larrán Jorge, M. \& Andrades Peña, F.J. (2015). La oferta de asignaturas de responsabilidad social corporativa y ética empresarial en las titulaciones de finanzas y contabilidad: Análisis comparativo con el ámbito de la gestión de organizaciones. Revista de contabilidad, 18(1) 1-10. doi: 10.1016/j.rcsar.2013.10.001

López Quintás, A. (2003). Inteligencia creativa: El descubrimiento personal de los valores. Madrid: Biblioteca De Autores Cristianos.

Reason, R. D., Ryder, A. J., \& Kee, C. (2013). Higher education's role in educating for personal and social responsibility: A review of existing Literature. New Directions for Higher Education, 164, 13-22. doi: 10.1002/he.20072

Robledo Ramón, P., Fidalgo Redondo, R., Arias Gundín, O., \& Álvarez Fernández, L. (2015). Percepción de los estudiantes sobre el desarrollo de competencias a través de diferentes metodologías activas. Revista de Investigación Educativa, 33(2), 369-383. doi: 10.6018/rie.33.2.201381

Sánchez González, C. L., Herrera Márquez, A. X., Zárate Moreno, L. G., \& Moreno Méndez, W. (2007). La responsabilidad social universitaria (RSU) en el contexto del cambio de educación superior. México: Universidad Autónoma De México. 


\section{Apéndice}

(Valores de 1 a 6 puntos, indicando el valor 1 mínimo acuerdo y el 6, máximo acuerdo)

\section{Cuestionario RSEU}

DIMENSION 1. Compromiso con los demás y el entorno

1. Tengo una visión global de la situación actual del mundo y soy consciente de la necesidad urgente de un desarrollo sostenible.

2. Esa toma de conciencia aumenta mi interés como universitario en contribuir a la mejora de mi entorno más cercano.

3. Me cuestiono cuál es mi posicionamiento personal ante las injusticias sociales, ante el dolor ajeno.

4. Pongo en práctica mi capacidad de servicio y compromiso con inmigrantes, discapacitados, indigentes, niños sin recursos, ancianos...

5. Considero que una de mis obligaciones como persona es ayudar a los demás, desde el compromiso social.

DIMENSIÓN 2. Descubrimiento personal de los valores

6. Creo que el compromiso social se fundamenta en el reconocimiento y respeto de la dignidad de toda persona.

7. Reconozco la necesidad de abrirme a los otros, de ponerme en su lugar y buscar el bien común, por encima de intereses individualistas.

8. Considero que el cambio personal es un paso previo y necesario para cambiar la realidad que me rodea.

9. He experimentado en primera persona la felicidad que conlleva el servicio y la solidaridad.

10. Considero que la experiencia de darse a los demás es provechosa para descubrir valores personales.

DIMENSIÓN 3. Formación de la responsabilidad social

11. Considero que el hecho de ser universitario ayuda a tomar conciencia de la importancia de la responsabilidad social.

12. He reflexionado sobre la importancia de no permanecer indiferente o ajeno ante lo que les sucede a los demás.

13. Creo que en la medida en que estudie y me prepare a fondo en la universidad, podré aportar más al cambio social.

14. La formación que recibiré en la Universidad contribuirá en la práctica, a que aumente mi grado de responsabilidad social.

15. Considero que la responsabilidad social es una competencia que se debe trabajar en la Universidad.

DIMENSIÓN 4. Planteamiento del ejercicio profesional desde el compromiso social

16. Me planteo el ejercicio de mi profesión futura con una vocación de servicio y orientación al bien común. 
17. Considero que el buen hacer profesional implica compromiso, trabajo en equipo, constancia, empatía, tolerancia, honradez y respeto.

18. Creo que mi realización personal y mi felicidad pasan por ser un profesional comprometido en la mejora del conjunto de la sociedad.

19. Considero que la acción de un buen profesional tiene repercusión en su entorno más inmediato y en otros de trascendencia mayor.

20. Creo que es realista afirmar que desde el ejercicio profesional es posible el compromiso social.

ÍTEM CRITERIO

21. A modo de síntesis, valora el grado en el que te sientes comprometido y socialmente responsable en estos momentos.

Fecha de recepción: 26/11/2015

Fecha de revisión: 03/12/2015

Fecha de aceptación: 04/04/2016 
